\title{
Can Benign Increased Intracranial Pressure cause Unilateral Papilledema? - A Case Report
}

\author{
Mohammed Attia* \\ Lecturer of Neurosurgery, Al-Azhar University, Cairo, Egypt \\ *Corresponding author: Mohammed Attia, Lecturer of Neurosurgery, Al-Azhar University, Cairo, Egypt. \\ To Cite This Article: Mohammed Attia, Can Benign Increased Intracranial Pressure cause Unilateral Papilledema? - A Case Report. 2020 - \\ 7(6). AJBSR.MS.ID.001212. DOI: 10.34297/AJBSR.2020.07.001212.
}

Received: 眥 February 20, 2020; Published: 制 March 05, 2020

\begin{abstract}
Benign Increased Intracranial Pressure: also called Idiopathic intracranial hypertension or Pseudotumor Cerebri is a condition in which there is elevation of cerebrospinal fluid pressure with unknown aetiology (so, idiopathic). This disorder usually affects women in childbearing period. With this disorder, there is No diagnosed exact pathology causing this problem. There are also no abnormalities in CSF pathways within the ventricles. Neuro-radiological diagnostic studies are also normal except those denoting high cerebrospinal fluid pressure. Also, there is no evidence of other secondary causes of increased intracranial pressure can be detected. We present a case of benign increased intracranial pressure in a young obese female presented with Unilateral papilledema and sudden unilateral deterioration of vision.
\end{abstract}

\section{Introduction}

Benign Increased intracranial pressure usually presents with bilateral papilledema. However, presenting by unilateral or highly asymmetric papilledema is a rare occurrence. Occurrence of this rare presentation may mislead treating physicians away from reaching the proper diagnosis and early help of patients to save their vision. The most common causes of Unilateral papilledema are local causes of the optic nerve or intraocular pathology.

\section{Case Report}

Female pt, 30 ys old, obese with body mass index 32.6, not known to have any previous systemic disease or history of trauma or other problem, developed acute severe headache affecting Lt side of the head, pulsating in character, associated with nausea, tinnitus of Lt ear, Lt ocular pain followed within few days by gradual rapidly progressive loss of vision of Lt eye until becoming only little perception of light and normal vision in Rt eye. The pt asked medical advice at Ophthalmology clinic before visiting a neurologist or neurosurgeon and informed that she has Unilateral Papilledema on Lt side and Normal Optic disc on Rt side and referred her to Neurosurgical consultation and for MRI Lt Orbit. There was no family history of autoimmune or neurological diseases. On examining the pt by a neurosurgeon after about 7 days of presentation, there was
Intact motor power, sensation and reflexes. There was marked loss of vision of Lt eye up to little perception of light, Dilated Lt pupil with very sluggish reaction, On eliciting light reflex, there is loss of direct light reflex on Lt side and preserved consensual light reflex on Lt side when directing light on Rt eye denoting Lt Optic nerve pathology. MRI Brain was asked for to detect any intracranial pathology and there was No SOLs with empty Sella sign. Orbital MRI with fat suppression and with contrast revealed Picture suggestive of Idiopathic intracranial hypertension. MRV Brain was also done and there was No abnormality detected. The pt was admitted to the Ward, started Steroid therapy on 9th day of presentation in the form of Prednisolone $50 \mathrm{mg}$ daily. Diagnostic lumbar puncture was done at OR under complete aseptic precautions and Opening CSF pressure was measured in recumbent Lt lateral position which was high (about $30 \mathrm{CmH20}$ ). Tapping of about $40 \mathrm{ml}$ of CSF was done until CSF flow became slow. CSF samples were collected for Physiology, chemistry, cytology and immunology. The pt was then shifted to the ward on medical ttt and IV fluid with close observation where the pt showed progressive improvement in visual acuity of Lt eye with the use of steroids and after lumbar puncture. The pt then was Discharged to home on medical ttt in the form of Acetazolamide, Furosemide, Prednisolone, Proton pump inhibitors 
and Neurotronic. The pt showed progressive improvement of visual acuity of Lt eye. On repeating Fundus examination, there is regression of papilledema. There is improvement of headache at all. CSF examination was within normal limits. Lab investigations done revealed no abnormality detected.

\section{Discussion}

Benign Increased Intracranial Pressure is a condition in which there is elevation of intracranial pressure with No clinical, radiological or laboratory evidence of hydrocephalus or any other intracranial pathology. To diagnose Benign increased intracranial pressure, it will be through the Modified Dandy Criteria that include Presence of symptoms and signs of high intracranial pressure including headache and papilledema, CSF Opening Pressure more than $25 \mathrm{Cm} \mathrm{H2O}$, Absence of localization signs apart from possible abducent nerve palsy, within normal CSF constituents, Normal or slit ventricles on imaging with absence of intracranial pathology, Absence of unexplained symptoms and signs, exclusion of intracranial venous sinus thrombosis by MR venography [1] It usually occurs in obese females in childbearing age period [2]. There is No clear etiology of benign increased intracranial pressure. However, it may occur due to alteration in one or more of the following: intrasagittal sinus pressure, decreased absorption of CSF by arachnoid villi, increased rate of CSF production, and compliance of CSF space. Occurrence in obese young women may be caused partially by raised CSF production [3-5]. Symptoms of intracranial hypertension include headache, pulsatile tinnitus, amaurosis fugax (brief loss of vision or blackouts) and loss of vision. Signs of intracranial hypertension include Diplopia if there is abducent nerve palsy or paresis, swelling of optic disc and visual loss [2]. Bilateral papilledema and absence of intracranial pathology including hydrocephalus are the common presentation of Benign increased intracranial pressure [6]. However, Unilateral papilledema is a rare occurrence with Benign increased Intracranial Pressure [7]. There are many case reports describing unilateral papilledema with benign increased intracranial pressure $[3,8,9]$. Sapkota et al. reported a case report of unilateral papilledema in a female pt aged $25 y s$ old and diagnosed as benign increased intracranial pressure [10]. King \& Floyd reported a case of Benign increased Intracranial pressure with Unilateral Papilledema [11]. In another case report by Brosh \& Strassman, there was unilateral papilledema in a female patient 25ys old with benign increased intracranial pressure [8]. The actual incidence of Unilateral papilledema with benign increased intracranial pressure is undetermined. Bruntse conducted a review study and found 25 out of 1346 patients with benign increased intracranial pressure had unilateral papilledema [12]. In another study conducted by Frederick, 6 out of 26 patients had unilateral or highly asymmetric papilledema [13]. The actual cause of occurrence of unilateral papilledema in benign increased intracranial pressure is still unknown. There are some hypotheses explaining the cause of unilateral papilledema. One of them supposed presence of anomaly of optic nerve sheath that may protect one optic nerve from the effect of intracranial hypertension. However, in a study conducted by Huna-Barn et al, there was 11 cases of unilateral papilledema and there was no evidence of differences between optic nerve sheaths on CT scan or MRI [3]. Another hypothesis postulated that there is a difference in lamina cribrosa of two optic discs [8] and this hypothesis was supported by another study that found axoplasmic blockage at the level of lamina cribrosa following experimental elevation of intracranial pressure [4].

\section{Conclusion}

Although rare, Unilateral papilledema can occur with Benign increased intracranial pressure. It should be kept in mind during differential diagnosis of unilateral papilledema and unilateral visual loss. Early diagnosis of these cases may help in avoidance of complications. We report this case to increase awareness that Unilateral papilledema may be a presentation of benign increased intracranial pressure.

\section{References}

1. Maloney K (2013) Idiopathic intracranial hypertension as an initial presentation of systemic lupus erythematosus. BMJ Case Rep 2013: Bcr2013010223.

2. Wall M (2008) Idiopathic intracranial hypertension (Pseudotumor cerebri). Curr Neurol Neurosci Rep 8(2): 87-93.

3. Huna-Baron R, Landau K, Rosenberg M, Warren FA, Kupersmith MJ (2001) Unilateral swollen disc due to increased intracranial pressure. Neurology 56(11): 1588-1590.

4. Tso MOM, Hayreh SS (1977) Optic disc edema in raised intracranial pressure. Arch Ophthalmol 95(8): 1458-1462.

5. Donaldson JO (1981) Pathogenesis of pseudotumor cerebri syndromes. Neurology 31(7): 877-880.

6. Bjerre P, Lindholm J, Gyldensted C (1982) Pseudotumor cerebri: A theory on etiology and pathogenesis. Acta Neurol Scand 66(4): 472-481.

7. Chari C, Rao NS (1991) Benign intracranial hypertension-Its unusual manifestations. Headache 31(9): 599-600.

8. Brosh K, Strassman I (2013) Unilateral papilledema in pseudotumor cerebri. Semin Ophthalmol 28(4): 242-243.

9. Sher NA, Wirtschafter J, Shapiro SK, See C, Shapiro I (1983) Unilateral papilledema in 'Benign' intracranial hypertension (Pseudotumor Cerebri). JAMA 250(17): 2346-2347.

10. Sapkota D, Singh S, Laurent B (2018) Unilateral Papilledema Due to Idiopathic Intracranial Hypertension-A Case Report. Med Case Rep 4(4): 87.

11. King W, Floyd AW (1990) Unilateral Papilledema in Pseudotumor Cerebri. Arch Ophthalmol. 108(5): 644-645.

12. Bruntse E (1970) Unilateral papilloedema in neurosurgical patients. Acta Ophthalmol 48: 759-764.

13. Lepore FE (1992) Unilateral and highly asymmetric papilledema in pseudotumor cerebri. Neurology 42(3): 676-678. 